Session 1432

\title{
Microelectronics-Photonics Interdisciplinary Science/Engineering Graduate Program Startup - Lessons Learned at the Five Year Point
}

\author{
Ken Vickers, Ron Foster, Greg Salamo \\ University of Arkansas
}

Background:

The University of Arkansas defined in 1998 an experimental interdisciplinary technology graduate program in Microelectronics-Photonics (microEP). While the microEP Graduate Program is an interdisciplinary degree-granting entity reporting directly to the Graduate School, its academic program elements are reviewed and approved through the normal academic processes of both the Fulbright College of Arts and Sciences and the College of Engineering. Faculty and students enter the program primarily with Physics, Chemistry, and Electrical, Chemical, and Mechanical Engineering backgrounds, but may enter from any rigorous science or engineering degree program. The first students entered the program in the fall 1998 semester, with the MS and PhD microEP degrees fully approved in July 1999 and July 2000 respectively.

The traditional research and educational focus of this program is electronically and photonically active materials, the devices that can be made from those materials, and the high performance solid systems that can be made from the combination of materials and devices. The nontraditional educational focus is in the management of the systems and human resources that move these technologies from the laboratory into full commercialization for the benefit of society. Specifically, the microEP graduate program strives to emulate an industrial work group in an academic environment, an environment that is based in assessing performance through evaluation of individual projects and knowledge rather than in meeting group objectives.

The microEP program also stresses the concepts of civic responsibility through the concept of the "citizen technologist". All microEP students are trained in their responsibilities to lead their communities after graduation to repay the large investment that society has placed into their graduate education. Inherent in this is the need to support the K-16 educational pipeline that will produce the next generation of their professional colleagues. It is important to lead through example, so the microEP faculty and administration have pursued resources to actively participate in all of these activities. The microEP program has received NSF IGERT, REU, RET, GK-12, and MRSEC awards; and a Department of Education FIPSE award to implement the microEP educational concepts in the traditional Physics Graduate Program.

The history of the microEP program formation, along with the details of its approach to graduate education, have been fully described in a paper presented by the authors at the 2002 ASEE Annual Convention ${ }^{1}$. The program has now produced over twenty-five MS graduates and three 
$\mathrm{PhD}$ graduates through August 2003, making it appropriate to review the performance of this experimental program at the five-year point of its existence. Particular emphasis will be placed on discussing the critical issues faced in administering an interdisciplinary program in a University system strongly based in traditional academic administrative structures.

Key microEP Program Elements:

In forming the microEP graduate program, a core tenet was that U.S. traditional science and engineering graduate programs are recognized world wide for their strength in giving their students excellent technical knowledge. The evidence of the power of these traditional graduate programs is in the high number of students from around the world who apply to the programs on a routine basis. The microEP program has maintained the full scope of academic methods in these traditional programs in transferring technical knowledge, while adding new methods and techniques to improve its students' ability to utilize that knowledge effectively in their early professional careers. These new methods and techniques include:

- Creating a natural work group of all students entering the program in one academic year (the Cohort).

- Creating an industrial style two-day creativity and innovation-training event at the end of each summer to enhance student expectations of themselves and to rapidly integrate new students into the microEP community (Summer Camp).

- Scheduling of routine external socialization events for continuing enhancement of the natural workgroup community.

- Introducing entrepreneurial and intrapreneurial skills through the creation of a specific course (MGMT 5383 Intra/entrepreneurism of Technology).

- Practicing entrepreneurial skills through application of theoretical knowledge in assessing current research for commercialization potential.

- Creating and/or improving student interpersonal skills and managerial skills through theory and practice in four semesters of MEPH 5811/6811 Operations Management.

- Creating a forum for focused discussions in the application of ethical behaviors in professional environments through a full summer seminar series MEPH 5821 Ethics for Scientists and Engineers.

- Creating a full summer course in funded program management that requires each student to submit a full funding proposal with his or her major professor to an appropriate agency at the end of the course (MEPH 5831 Proposal Writing and Management).

- Requiring each student to use Microsoft Project ${ }^{\mathrm{TM}}$ to plan their research activities with monthly reporting of Project Gantt charts.

- Creating a program management structure that embraces existing legacy structures while creating new structures to support specific interdisciplinary needs.

- Creating curriculum boundary conditions that well define the program's academic and research focus while allowing students maximum flexibility to define curricula to support their career ambitions.

These methods and techniques are not innovative when taken individually, and in fact individual methods can be found widely adopted in industry and academics. The innovation of the microEP 
Graduate Program is in the rigorous implementation of this total combination in the academic environment.

This rigorous implementation fosters management skills development without intruding on the academic hours needed for academic courses in technology. The glue that binds these techniques together is the simple, yet profound, technique of creating a community of students with a common goal of maximizing the academic success of each student in that community. The creation of this Cohort community, rather than having just a group of students in a common set of academic classrooms and laboratories, is properly described as the heart and soul of the microEP graduate program.

Each of these methods will be described in more detail, along with their effectiveness in implementation and planned modifications to be implemented as the program evolves and matures.

The Cohort natural work group:

The Cohort is the home community of all microEP students, whether they join the program as MS students directly after their BS degree is completed or if they join as a PhD student after their completion of a MS degree from a traditional graduate program. The students in a cohort face common barriers to academic success:

- Culture shock of moving to a new community (and sometimes country)

- Lack of knowledge of institutional resources and infrastructure methods

- Lack of knowledge of operational expectations of faculty in the classroom

- Lack of knowledge of operational expectations of faculty in the laboratory

- Lack of academic knowledge in a new area of graduate academic coursework

By using proven industrial team-building techniques, students are brought together first as a new Cohort and given an opportunity to know each other. The new Cohort's members are then brought together with the rest of the microEP community of students in a way that they are able to learn individuals from all other Cohorts. Part of these activities includes utilization of competitive events between cohorts in creativity exercises.

The primary outcome of all community-building activities is to build a common culture and expectation of trust between all microEP students. It is this common culture of trust, respect, and mutual support that allows the microEP program to require that students have a responsibility to assure that every student achieves the highest level of academic success of which they are capable.

As the barriers to academic success listed above span everything from culture to academic knowledge, all students must trust their colleagues to effectively overcome the barriers. This trust only comes from being part of a community instead of being part of a group. Creating community trust is the core value of the microEP program, a task that immediately focuses the student in an outward direction instead of the inward focus of most academic programs. It also forces the entering student to begin thinking in terms of group dynamics and group optimization. 
A key insight that is quickly gained by the students is that the most difficult task for them will not be in tutoring a fellow Cohort member who is having academic problems, but is instead trusting his Cohort colleagues enough to ask for help when his own academic performance is declining.

Summer Camp - a high intensity community formation event:

Most students enter graduate programs during the fall semester, with some students reporting to campus during the summer and a few students joining a program in the spring semester. All summer and fall entry students in a new Cohort are required to report to campus one week before the start of the fall semester. During this week, traditional orientation activities occur to logistically introduce the students to the campus infrastructure.

The event that is not typical during this week is an industrial style two-day "summer camp" to immerse the students in a physically active retreat. The microEP program started with the creativity training concepts developed by Dr. Ed Sobey (www.inventioncenter.com) and modified them to include additional emphases on community development. The activities during the retreat combine elements of creativity training, team building, awareness expansion, etc. The camp is required of all program students, both the entering Cohort members and the returning Cohorts.

Activities are chosen that require both small and large group cooperation, and some activities are designed to foster a good-natured competitiveness between the Cohorts while growing the since of identity with the graduate program as a whole. The camp resembles at times a toy factory in an interior classroom environment, and then transforms itself into a ropes course-type outdoor activity with students being manhandled through a spider-web like rope maze.

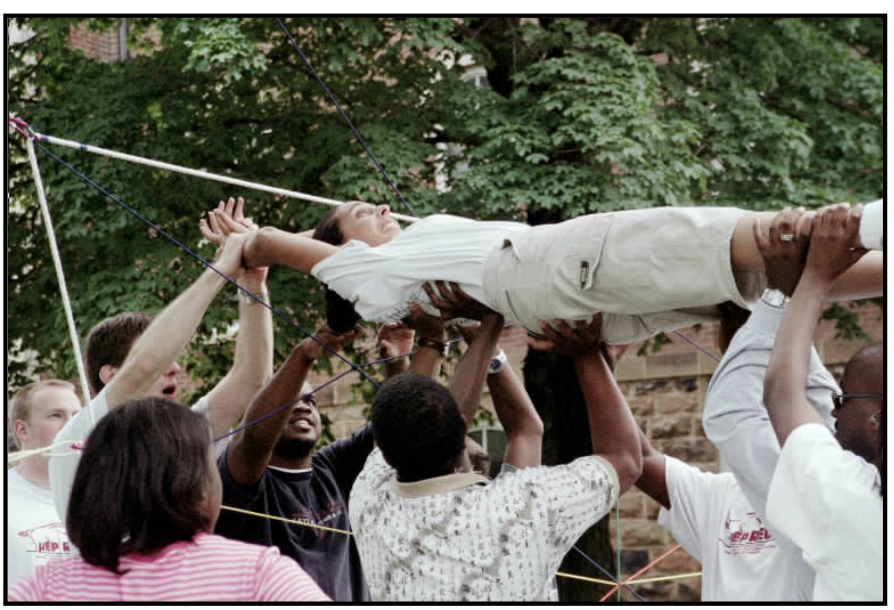

Figure 1: The Spider Web activity

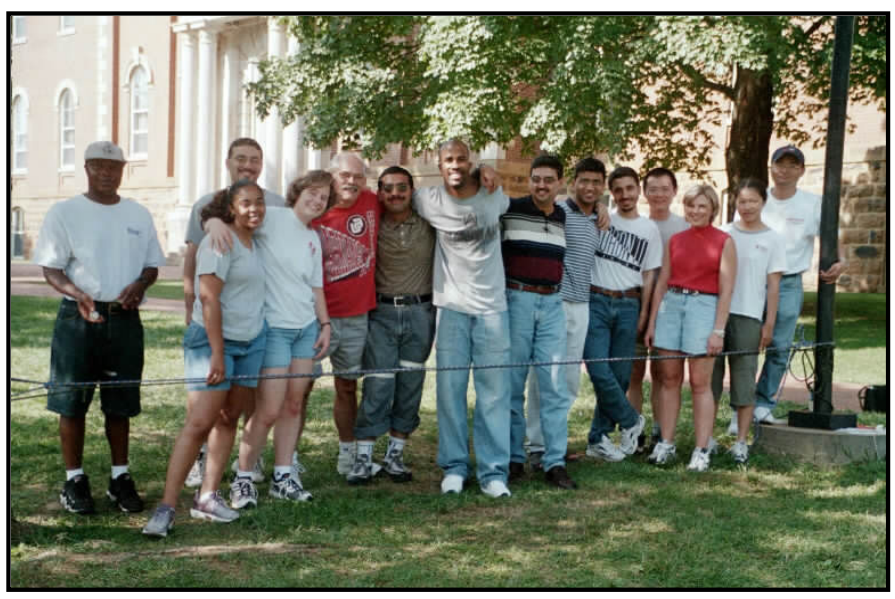

Figure 2: Success in the Electric Fence activity

The result of this two-day activity is easily seen in the Cohort versus Cohort competitive events late in the afternoon of the second day. The new Cohort's members have established basic group cooperation skills by this time, but the older Cohorts' members are fully coordinated and 
supportive. It is most easily observed in activities that may require physical activity that is difficult for certain individuals, which any person can voluntarily recluse themselves from participation. In the new Cohort, the group allows students to recluse themselves if they are hesitant about the exercise. In the older Cohorts, the group will always find a way to overcome physical limitations of members to keep them involved in the exercise.

\section{External Socialization Events:}

The creation of effective natural work groups is enhanced by socialization events external to the work place $^{2}$. The microEP Graduate Program creates many different types of opportunities for socialization, including:

- Group dinners organized around U.S. cultural events such as Halloween (Jack-o-Lantern carving contest), Thanksgiving, Easter, etc., as well as during non-holiday months.

- Group trips to cultural or regional natural attractions (canoe trip on the Buffalo River, shopping at Eureka Springs, hiking in the Ozark National Forest, etc).

These events are purely voluntary, with participation or non-participation not affecting the students' status in the program in any fashion. The most

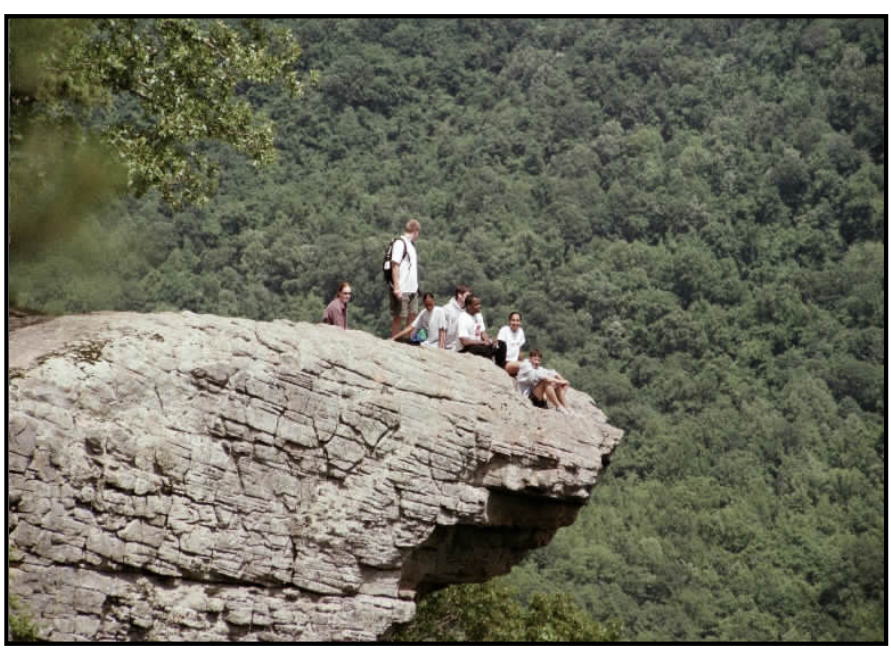

Figure 3: Group hike into Ozark Forest difficult aspect of this technique is the high level of energy and time required of program management and faculty, as well as the difficulty in creating a wide variety of events from which the students may choose.

Creating Entrepreneurial Skills:

This technique addresses the fact that technology based graduate students will typically try to accomplish anything, provided that they understand their level of knowledge and what they must learn in order to be successful.

In traditional science/engineering graduate programs students are not given any academic preparation or any practice in entrepreneurship. This lack of knowledge, including not even understanding the scope of what they don't know, creates an emotional barrier to many technology students when considering commercialization prospects of the intellectual property created in their own research.

To overcome this shortcoming of traditional programs, the microEP graduate program won financial support from the National Collegiate Inventors and Innovators Alliance (www.nciia.org) to create a course co-taught by Prof. Vickers and Dr. John Todd of the Walton 
College of Business. This course was specifically designed to teach both technology and business students the exact knowledge that they lack that would allow them to successfully commercialize research in rapidly developing high technology industrial segments. The course, MGMT 5383 Intra/Entrepreneurism of Technology, has been very successful in producing both spin-off technology companies by its graduates and in receiving national recognition for innovative instruction ${ }^{3}$.

Practicing Entrepreneurial Skills:

Students in MGMT 5383 are given the opportunity to practice their skills by engaging with a university researcher that has begun the intellectual property protection process by submitting patent disclosures to the Technology Transfer office. Students sign nondisclosure agreements with the University concerning the research topic of a volunteer inventor, and then apply the knowledge they have gained at each step of the course to that invention.

This gives the students an emotional understanding of the commercialization process, both in understanding its difficulty as well as understanding that it can be successfully accomplished with hard work and proper development team formation.

The results of this theory and practice of entrepreneurial skills has far exceeded our expectations, especially in terms of changes in the culture of the research labs on our campus. We expected that our students would be more likely to consider commercialization of their own research, and two of our PhD graduates have started their own spin-off companies that have already won one or more SBIR Phase I grants.

What we did not realize was that for five years we have been loading faculty research groups around the campus with "Trojan Horse" graduate students - students that are demanding that

Figure 4: NSF SBIR Awards by Year in Arkansas

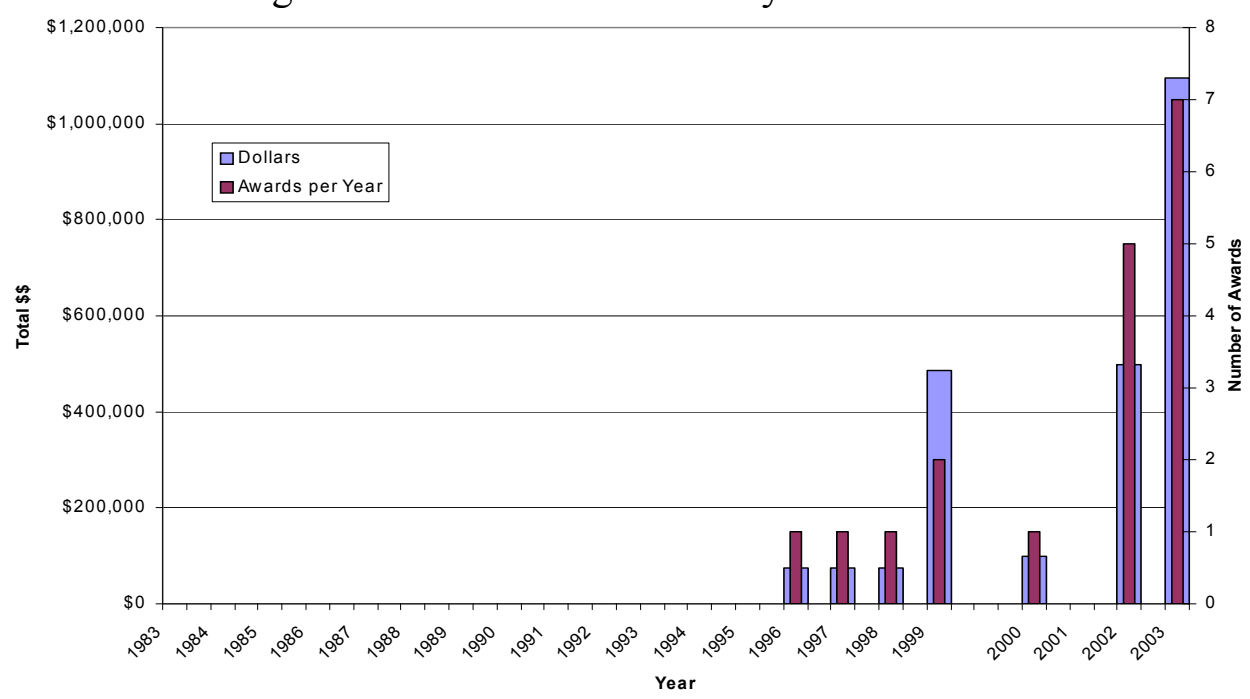

their hard work in research bring more reward than just the academic publications. This has been one of the key elements of a mosaic of entrepreneurial support activities on our campus, 
together causing a more rapid change in faculty attitudes toward research commercialization than we thought possible.

An overview of this effort has already been reported ${ }^{4}$ at the National Collegiate Inventors and Innovators Alliance (NCIIA) annual meeting in San Jose, California in March 2004. Figure 4 is drawn from that presentation, and demonstrates the sharp increase in NSF SBIR activity in Arkansas (with almost all of the activity centered in the University of Arkansas campus). What is not demonstrated in Figure 4 is that all 2003 SBIR grants on the U of A campus were won by microEP graduate program faculty and students, providing additional evidence that the microEP graduate program's methods have been a key element in changing the entrepreneurial culture on our campus.

\section{Practicing Interpersonal/Management Skills:}

The Cohort group identity is strong at the end of the summer camp, but would be subject to rapid decay if it were not refreshed through a weekly operations seminar of each Cohort group. In these seminars, discussion topics include issues that affect a person's operational effectiveness in a technology-based workplace. The subject matter of each meeting is structured to address the level of organizational and interpersonal skills training that has already been received by the students.

Students in these seminars also have a chance to learn in detail how other world cultures address educational issues, social interactions, family responsibilities, etc. Through frank discussions with Cohort colleagues that they know personally (and trust because of that knowledge), many preconceptions about these other cultures can be examined and understood. This results not in agreement on what are the merits of the different cultural approaches, but rather that working with professionals from other cultures can easily lead to communications difficulties - even when both sides of a conversation are trying hard to cooperate. This is in a real sense a vaccination in cultural tolerance, a tolerance that will be highly needed if the student is working in a worldwide technical environment.

These seminars have proven to be highly effective and are continuing in much the same format as when first started in 1998. What has evolved is a progression of topics in a set of linked syllabi that is normally followed; unless local or world events provide an opportunity to discuss the training they are receiving in a "live fire" exercise as events unfold in time.

\section{Practicing Ethical Decision Making Skills:}

Today's students have matured in a world in which core cultural, moral, and ethical values are constantly challenged and modified. The worldwide rate of piracy of electronically based intellectual property is a well-discussed example of changing attitudes toward theft.

During the summer, microEP students meet weekly in MEPH 5821 Ethics for Scientists for Engineers to discuss the application of ethical decision making in professional situations. The class uses the book Ethics in Engineering Practice and Research by Whitbeck, reading one chapter per week and discussing that chapter in the once per week two-hour class period. 
This summer class provides specific content knowledge and practice in consideration of responses to ethical dilemmas, but also provides an important opportunity for the students to meet with program management on a weekly basis. This has proven to be very effective in giving students new insights into their own decision making process, and has also prevented microEP students from sliding into a non-effective use of their summer through lack of visibility to program management.

This class is also taken by undergraduates participating in the microEP hosted NSF Research Experience for Undergraduates (REU) site. These students are typically rising juniors and seniors, and are among the top students in our country's undergraduate programs. As part of the REU program evaluation, the REU students each year have been given a survey in which the self-assess their practice of ethics in general.

The students assess themselves on a five-point scale from 1 (poor ethical practices) to 5 (highly ethical practices). They are then asked to self assess any changes in their ethical practices as a result of the course, only now the answers are separated into personal ethical practices and professional ethical practices. Once again they use a five-point scale from 1 (no change) to 5 (great change). The results for the summer 2003 REU students are shown in Figures 5 and 6.

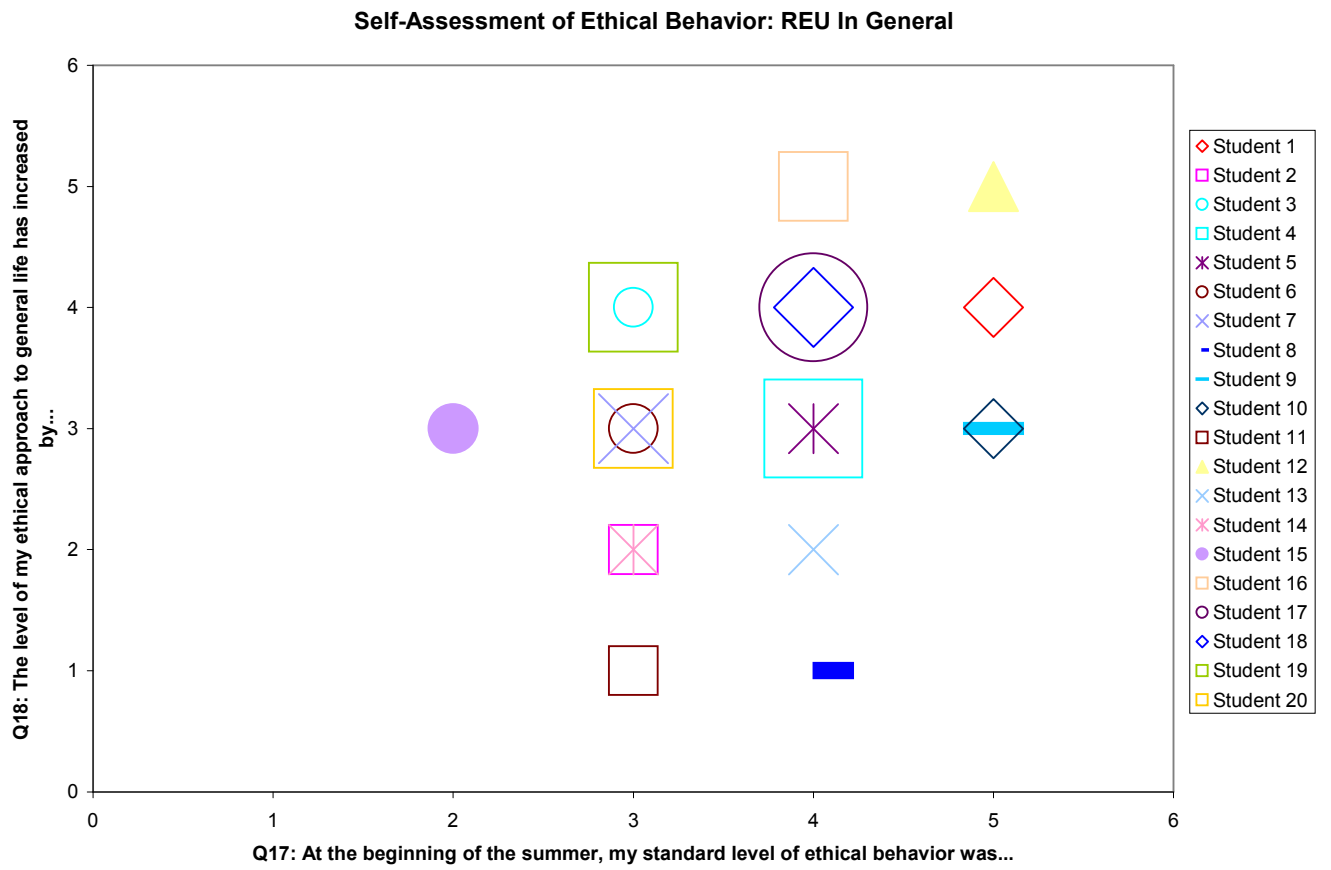

Figure 5: Improvement in personal ethical behavior

Figure 5 clearly demonstrates that the students almost all saw themselves as having average to high ethical behaviors at the beginning of the summer class. What is interesting in the data is that every student except two saw the REU student group as a whole as improving their personal ethical behavior during the course of the summer. 
Examination of Figure 6 shows a similar trend in the improvement of professional ethics. Only in this case, the students' practice of professional ethics for the group is shown as sharply increased. All students, regardless of their beginning self-assessment, felt that the summer class in ethics had improved their practice of professional ethics - with almost all students indicating moderate to very high changes. Extensive follow-up interviews with the students were not made, but anecdotal comments taken from the surveys indicate that this summer course was their first opportunity to test their personal ideas against the type of professional challenges represented by the case studies in the textbook.

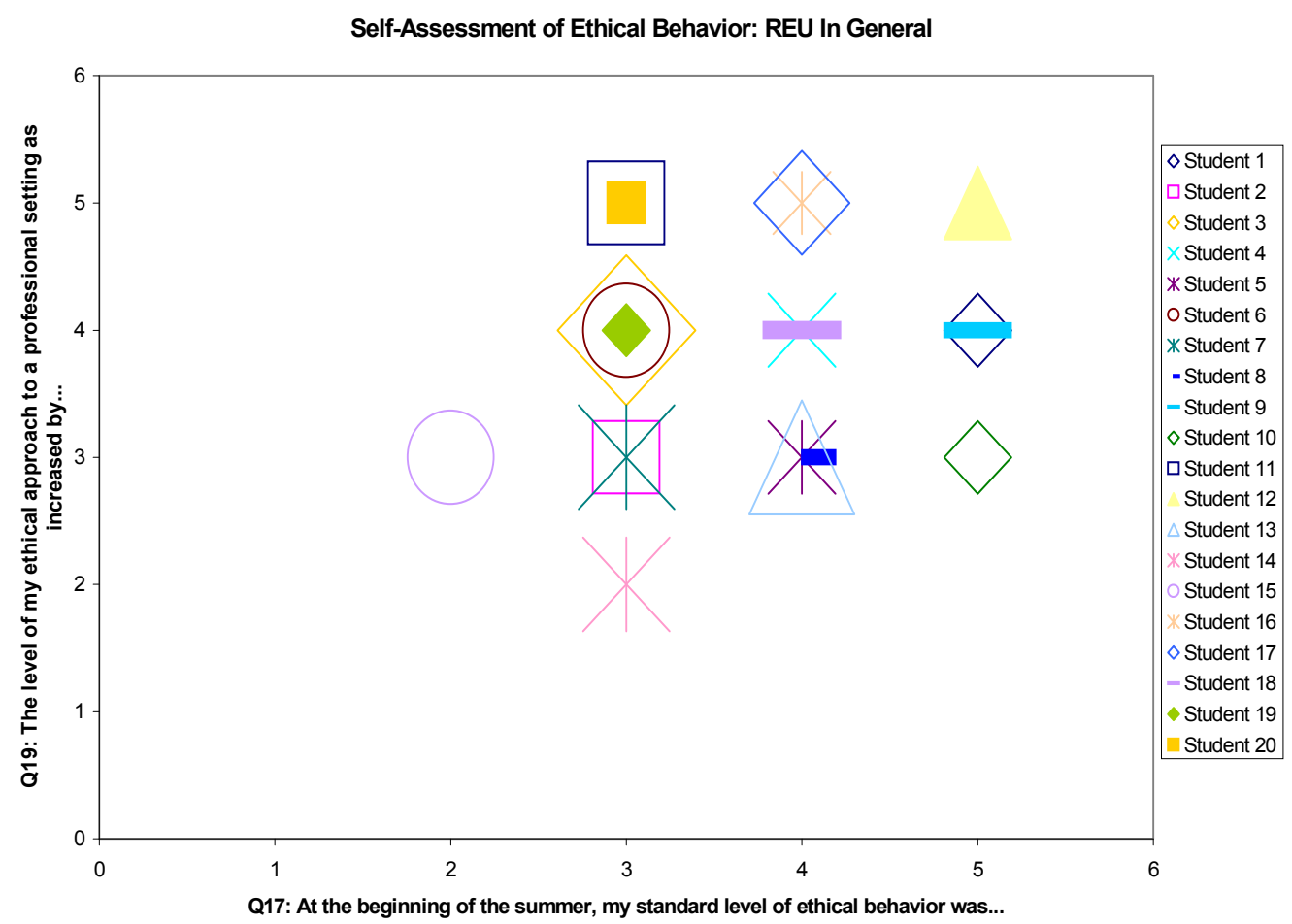

Figure 6: Improvement in professional ethical behavior

This survey has not been given to the graduate students in the class, but that will be done at the beginning with the summer 2004 class. From conversations with graduate students who have taken the class, we do not anticipate that the results will be significantly different than the results from the undergraduate student surveys. We do feel that this course is having a significant impact on the preparation of microEP students for their professional careers.

Developing Project Development and Management Skills:

Graduate programs do not generally focus on engaging their graduate student populations in creating the programs that provide their own funding. The microEP program feels that this is a vast un-tapped source of innovation and creativity to expand research funding and opportunities.

The summer course MEPH 5831 Proposal Writing and Management was designed specifically to engage students in creating funding opportunities for themselves and their research laboratories. 
Students are trained in both the methods and logistics of proposal writing, as well as in the management of proposals after they are funded.

Students work with their major professors to identify new solicitations that fit into the scope of the professor's research laboratory, but would expand the laboratory into new directions. During the course of the summer the student will write a complete proposal that is reviewed by the course instructor and the other students. After the course is completed, the professor works with the student and other collaborators to polish the proposal and submit it to the funding agency.

Even if the proposal is not funded, the graduate students are graphically introduced to the level of effort needed to create funded research. They become more efficient at utilizing their own research resources, and are usually much more appreciative of the funding they receive in research assistant positions.

By meeting once per week the students have sufficient opportunity for writing the proposal without shutting down their summer research. Meeting weekly also gives the microEP management team the same opportunity to interact with the students that has been described in the MEPH 5821 ethics course. The class is taught every summer, and has even been taught in a short course format to faculty and community members through the continuing education office.

Research Planning using Microsoft Project ${ }^{\mathrm{TM}}$ :

Science graduate programs, and even some engineering graduate programs, have a negative response to the role of project planning in university-based research. Comments such as "you can't plan breakthroughs" and "Albert Einstein would never have used this" are often heard among faculty being introduced to project planning.

The key to faculty acceptance is in gaining recognition that while you cannot schedule creative events, you can certainly plan how you are going to investigate the ideas that occur when creativity does strike.

Microsoft Project ${ }^{\mathrm{TM}}$ is widely used in industry, with microEP alumni reporting that about one third of them use it routinely, one third may use it and have it available on their workstations, and one third rarely see it in their workplace. The microEP program utilizes this particular software because of the breadth of availability, but focuses the educational efforts on the project planning process itself.

\section{Program Management Structure:}

The microEP program was designed to leverage educational and research interests already in existence among the faculty as the program was created. It was designed to be a supplementary partner to the traditional departmental programs instead of a competitor to the programs. Its goal was to attract new students to the campus to work in advancing the research efforts of the faculty, students who would not have chosen to enroll at this university without the existence of the microEP program. 
One aspect of this partnership is that a student who graduates with a microEP degree is seen as a graduate of both the microEP graduate program and of the department of the students' major professor. The microEP program has also aggressively pursued educational funding opportunities that have provided faculty with top quality funded graduate students and with supported opportunities to create new course content.

The microEP faculty group first approves all academic program elements, with the group comprised of faculty members who voluntarily associate themselves with microEP while being appointed to traditional departments. After approval by microEP faculty, academic program elements go through the full approval process of both the Fulbright College of Arts and Sciences and the College of Engineering. No academic element is implemented unless fully approved by both Colleges and the Graduate School. In this fashion, microEP elements receive a higher level of review and approval than any traditional departmental element.

In a similar fashion, the administrative home of the microEP program is in the Graduate School instead of either College. While this has caused some amount of difficulty in administrative structure, it has been an essential element of validating that the microEP program is a true interdisciplinary program.

Creating Curriculum Boundaries:

The microEP program is defined as a professional development type graduate degree program, and its educational focus is considered inappropriately narrow for an undergraduate degree program. But at the graduate level this definition gives the microEP program the freedom to utilize any courses on campus that would comprise an appropriate collection of knowledge that is useful to a graduate student in the program.

This freedom of selection comes at the price of a lack of a generally accepted public knowledge of what can be expected of a microEP graduate. In the first years of the program the only core courses required of all students were those already described in management skills, ethics, and entrepreneurism. At the meeting of the microEP Industrial Advisory Committee meeting in October 2002, this lack of core curriculum was the single large constructive criticism of the program.

The microEP program has now implemented a core of one course in each of the four major emphasis areas of the program: Photonics, microelectronics, materials and processing, and entrepreneurial and management skills. This new core has been fully implemented with the entering students in Fall 2003, and would result in only minor curriculum modifications if implemented in all current microEP students.

Market acceptance of the MS microEP students has been very good, as most MS graduates go to an industrial career and this program stresses skills and knowledge that are highly valued by industry.

Surveys of University of Arkansas faculty as late as Spring 2002 have demonstrated a concern that academic departments would not value the interdisciplinary knowledge and skills of PhD 
microEP students, even though it was believed that industry would value them. However, starting in December 2003 there have been four PhD microEP graduates. Two have been hired into traditional academic post-doc positions on the basis of their broad skill sets, one has been directly hired as a teaching and research faculty because of his experience in both entrepreneurism and interdisciplinary education, and the fourth has now won three SBIR Phase I grants and one unsolicited grant in support of his local startup company based on his $\mathrm{PhD}$ research.

Summary:

The microEP graduate program has proven to be a very successful and popular graduate program at the University of Arkansas. Forty-nine students are enrolled and working on their graduate degrees in the spring 2004 semester. The number of interdisciplinary degree programs on the University of Arkansas campus has grown from one to four since the microEP implementation in 1998.

The authors believe that the microEP graduate program provides a model that can be duplicated at other institutions and welcome inquiries on how this program could be modified for implementation at other institutions.

\footnotetext{
${ }^{1}$ Ken Vickers, Greg Salamo; American Society for Engineering Education Annual Conference, June 2002; "Graduate Student Practice of Technology Management: The Cohort Approach to Structuring Graduate Programs"

${ }^{2}$ Ralph Katz, "The Human Side of Managing Technological Innovation, $2^{\text {nd }}$ Edition”, 2004, P34.

${ }^{3}$ Decision Sciences Institute, 2001 Annual Award for Instructional Innovation, http://www.decsionsciences.org.

${ }^{4}$ Ken Vickers, Ron Foster; National Collegiate Inventors and Innovators Alliance, March 2004; "Invited Panelist, NSF Partnership for Innovation Grantees"
}

\begin{abstract}
KEN VICKERS
Ken Vickers is a Research Professor in Physics at the University of Arkansas, and has served as Director of the interdisciplinary Microelectronics-Photonics Graduate Program since April 1998. He worked for Texas Instruments from 1977 through March 1998 in integrated circuit fabrication engineering, and has authored twenty-nine issued patents. He received BS and MS degrees in Physics from the University of Arkansas in 1976 and 1978 respectively.

\section{RON FOSTER}

Ron Foster is a Research Associate Professor at the University of Arkansas, and has served as Director of the Innovation Incubator (a NSF Partnership for Innovation sponsored activity) at the University since 2001. He worked for both Texas Instruments and Honeywell, and was engineering manager of both design and manufacturing of sensors. He received a BA Physics in 1977 and MS Physics in 1980 from the University of Arkansas.
\end{abstract}

\title{
GREG SALAMO
}

Greg Salamo is a University Professor of Physics at the University of Arkansas. Dr. Salamo received a BS degree in Physics from Brooklyn College in 1966, an MS degree in Solid State from Purdue University in 1968, and his Ph.D. in Optics from CUNY/Bell Labs in 1973. After a Post-Doc position at the University of Rochester, he joined the faculty of the University of Arkansas in 1975. 